Results Overall the diagnostic activity for HCV has increased over the last two decades. More markedly since 2012 when DAAs were becoming available. The standard diagnostic pathways (primary and secondary care) show large volume testing with a low rate of PCR positivity. In contrast testing pathways aimed at high risk individuals show a higher PCR positive rate. See figure.

Conclusions Utilisation of diagnostic pathways targeting populations most at risk of $\mathrm{HCV}$ are more effective at yielding new HCV diagnoses than standard pathways. These tailored diagnostic pathways will also resolve some of the health inequalities around drug use and provide methods of ensuring entry to treatment. We believe using targeted testing will find the majority of our undiagnosed population. This will help us to direct resources and achieve our aim of elimination by 2030.

\section{PTU-034 SPLEEN STIFFNESS BY ELASTPQ POINT SHEAR WAVE ELASTOGRAPHY PREDICTS CLINICALLY SIGNIFICANT PORTAL HYPERTENSION IN PBC}

\footnotetext{
1,2Francesca Saffioti" ${ }^{*}$ 'Davide Roccarina, ${ }^{1}$ Matteo Rosselli, ${ }^{1,3}$ Roberta Stupia, ${ }^{1}$ Aileen Marshall, ${ }^{1}$ Massimo Pinzani, ${ }^{1}$ Douglas Thorburn. ${ }^{\prime}$ Sheila Sherlock Liver Centre, Royal Free London NHS Foundation Trust and UCL Institute for Liver and Digestive Health, University College of London, London, UK; ${ }^{2}$ Department of Clinical and Experimental Medicine, University of Messina, Messina, Italy; ${ }^{3}$ Division of Clinical and Molecular Hepatology, University Hospital of Messina, Messina, Italy
}

\subsection{6/gutjnl-2019-BSGAbstracts.243}

Introduction There is an ongoing effort to identify non-invasive surrogates for staging liver disease and detecting clinically significant portal hypertension (CSPH), defined as the presence of gastro-oesophageal varices on endoscopy. The correlation of spleen stiffness measurement (SSM) with portal hypertension measured by hepatic venous pressure gradient has been reported, but data regarding its reliability are controversial.

We assessed the ability of liver stiffness measurement (LSM) and SSM performed by point-shear wave elastography (pSWE) to detect $\mathrm{CSPH}$ in primary biliary cholangitis (PBC).

Methods Predictors of CSPH were evaluated in $53 \mathrm{PBC}$ patients who had an upper-GI endoscopy within 12 months of the elastographic assessment. Demographics, biochemical and ultrasonographic data were prospectively collected. Transient elastography (Fibroscan, Echosens) [F-TE], liver and spleen pSWE (ElastPQ, Philips Affiniti70G), and the most common clinical fibrosis scores were obtained. ROC curves were constructed to establish the performance of elastographic techniques and fibrosis scores in predicting CSPH.

Results Mean age $57 \pm 12$ years, 91\% female. OVs were detected in 12 (23\%) cases (4 at high-risk of bleeding).

Variables significantly associated with CSPH at univariate analysis were: diagnosis of cirrhosis, Child-Pugh, MELD and Mayo risk score, spleen area and longitudinal diameter, platelets, albumin, ALT, INR, F-TE, ElastPQ LSM and SSM, LSPS (LSM*spleen diameter/platelets). SSM remained the only independent predictor of CSPH in all the multivariate models (OR 1.101; 95\%CI 1.14-1.20; $\mathrm{p}<0.022$ ).

F-TE and ElastPQ LSM showed good AUROCs (95\%CI): $0.85(0.72-0.99)$ and $0.80(0.62-0.98)$, with best cut-off 14.3 and $13.8 \mathrm{kPa}$, respectively. However, specificity $(\mathrm{Sp})$ was $<70 \%$ for both tests. ElastPQ SSM showed the best performance in predicting CSPH (AUROC 0.95, 95\%CI 0.87-1.00, sensitivity (Se) $88 \%$, Sp 97\%), with optimal cut-off $50.1 \mathrm{kPa}$
(PPV 0.89, NPV 0.97), followed by GUCI, King's score and LSPS [AUROC (95\%CI): 0.94 (0.87-1.00), 0.94 (0.85-1.00), 0.93 (0.85-1.00), respectively] (fig. 1).

In this cohort, the diagnostic performance of ElastPQ SSM in detecting CSPH was superior to the recently validated Baveno VI and Expanded Baveno VI criteria, which showed good Sp (77\% and 87\%, respectively) but low Se $(67 \%$, for both).

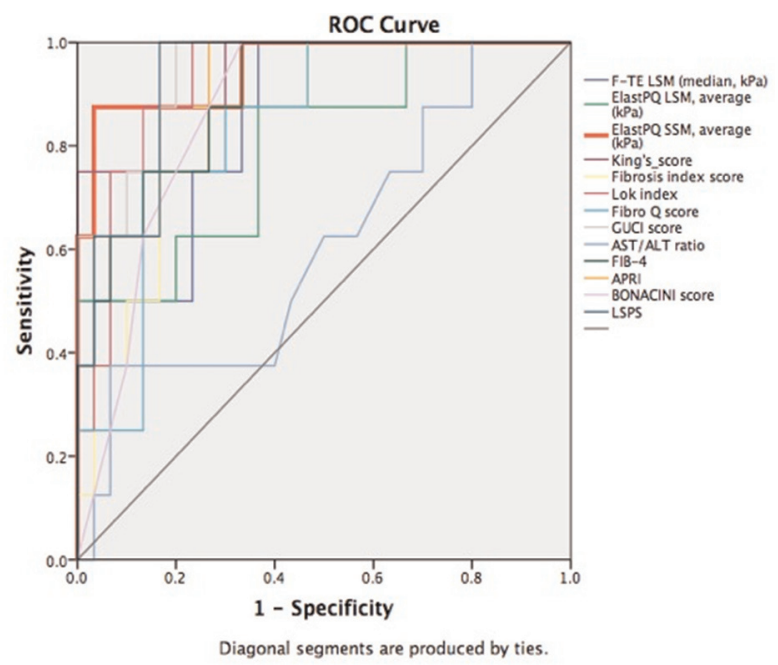

Abstract PTU-034 Figure 1

Conclusions We provide evidence that transient elastography, in particular ElastPQ SSM, can be used as a reliable tool for the detection of CSPH in PBC.

\section{PTU-035 ECONOMIC IMPACT OF NON-RESPONSE TO URSODEOXYCHOLIC ACID IN PRIMARY BILIARY CHOLANGITIS PATIENTS}

${ }^{1}$ Parivrudh Sharma*, 2 Lynne Crighton, ${ }^{3}$ Balasubramaniam Vijayan, ${ }^{3}$ Shirley English, ${ }^{3}$ Ashis Mukhopadhya. 'University of Aberdeen, Aberdeen; ${ }^{2}$ Pharmacy Department, Aberdeen Royal Infirmary, Aberdeen; ${ }^{3}$, Department of Digestive Disorders, Aberdeen Royal Infirmary, Aberdeen

\subsection{6/gutjnl-2019-BSGAbstracts.244}

Introduction NICE recommends the use of Obeticholic acid (OCA), as a second-line treatment for failed or intolerant ursodeoxycholic acid (UDCA) drug therapy in primary biliary cholangitis (PBC). This audit aims to determine the proportion of PBC patients, in a tertiary referral hospital experiencing failed UDCA drug therapy, to gauge the potential economic impact of a switch to OCA.

Methods A total of 120 patients with PBC were identified from an existing patient database. 24 patients were excluded due to inappropriate diagnosis, missing data, non-attendance or failure to tolerate UDCA. Baseline characteristics, UDCA dosage and biochemical response were recorded for all patients. For the purpose of this study, failed UDCA drug therapy was defined as an alkaline phosphatase (ALP) level of greater than 1.67 times the upper limit of normal (ULN) (Toronto Criteria) ${ }^{1}$.

Results Of the 96 patients included for analysis, 9 were male and the remaining 87 were female. The mean age and weight 